\title{
ON RIGHT DUO P.P. RINGS
}

\author{
by A. W. CHATTERS and WEIMIN XUE
}

(Received 21 November, 1988; revised 1 February 1989)

Throughout the paper, rings are associative rings with identity. A ring is called right duo if every right ideal is two-sided, and it is called right p.p. if every principal right ideal is projective. A left duo (p.p.) ring is defined similarly, and a duo (p.p.) ring will mean a ring which is both right and left duo (p.p.). There is a right p.p. ring that is not left p.p. (see Chase [2]). Small [9] proved that right p.p. implies left p.p. if there are no infinite sets of orthogonal idempotents, and Endo [5, Proposition 2] has shown the same implication in the case where each idempotent in the ring is central. Since Courter [3, Theorem 1.3] noted that every idempotent in a right duo ring is central, we can simply speak of right duo p.p. rings. A typical example of a right duo ring which is not left duo is the following. Let $F$ be a field and $F(x)$ the field of rational functions over $F$. Let $R=F(x) \times F(x)$ as an additive group and define the multiplication as follows:

$$
\left(f_{1}(x), g_{1}(x)\right)\left(f_{2}(x), g_{2}(x)\right)=\left(f_{1}(x) f_{2}(x), f_{1}\left(x^{2}\right) g_{2}(x)+g_{1}(x) f_{2}(x)\right) .
$$

Then $R$ is a local artinian ring with $c\left(R_{R}\right)=2$ and $c\left({ }_{R} R\right)=3$. Thus $R$ is right duo but not left duo.

Vasconcelos [13, Theorem 4.2] proved that a commutative ring $R$ is semihereditary if and only if $R$ is p.p. and the weak dimension $\mathrm{wD}(R)$ of $R$ is at most one. Recently Tuganbaev [12, Proposition 3] generalized Camillo's result [1] by showing that a duo ring is both right and left semihereditary if each two-generated ideal is right projective. It should be noted that Camillo's theorem [1] was a generalization of a much older result. Jensen [6, Lemma 3] claims that Dedekind [4] essentially proved a commutative integral domain is a Prüfer ring provided that every two-generated ideal is projective.

In this paper, we shall establish the following results.

THEOREM 1. Let $R$ be a right duo ring. The following statements are equivalent:

(1) $R$ is right semihereditary;

(2) every two-generated ideal is right projective;

(3) $R$ is p.p. and $\mathrm{wD}(R) \leq 1$.

THEOREM 2. Let $R$ be a duo p.p. ring. If I is a finitely generated right projective ideal then $I$ is left projective and a direct summand of an invertible ideal.

A ring with no non-zero nilpotent elements is called reduced. Our results are based on the following key lemma. We note that the right-duo assumption of $R$ in the lemma is essential: let $R$ be the ring of 2 by 2 upper triangular matrices over a field; then $R$ is artinian hereditary indecomposable but not semiprime, and its quotient ring is $R$ itself which is not von Neumann regular.

LEMMA 3. Let $R$ be a right duo p.p. ring. Then

(1) $R$ is reduced and has a right classical quotient ring $Q$ that is von Neumann regular;

(2) if $I$ is a right (projective) ideal that is $n$-generated i.e. $I=\sum_{i=1}^{n} x_{i} R, I$ is a direct summand of a n-generated essential right (projective) ideal.

Glasgow Math. J. 32 (1990) 221-225. 
Proof. (1) Let $K$ be a nilpotent ideal. The right annihilator $r(K)$ of $K$ is essential as a left ideal, for if $L$ is a non-zero left ideal then there is an integer $i$ such that $K^{i} L \neq 0$ and $K^{i+1} L=0$. Hence $r(K)$ is essential as a right ideal since $R$ is right duo. But $K \cdot r(K)=0$. Therefore $K=0$ because $R$ is right non-singular. Thus $R$ is semiprime. Suppose that $a^{2}=0$. Then $(a R)^{2} \subseteq a^{2} R=0$. Hence $a=0$. Thus $R$ is reduced.

Let $a \in R$. We have $r(a)=e R$ for some idempotent $e$ that is central by [3]. Since $a R \cap e R=0$, we have $a R+e R=(a+e) R$ and $a+e$ is regular (i.e. not a zero divisor). Hence $a R+e R$ is essential. The elements of the form $a+e$ as above are regular, and every regular element $c$ is of this form with $c=a+e$, where $a=c$ and $e=0$. Because $c R \supseteq R c$ for every regular element $c$, we know that $R$ satisfies the right Ore condition with respect to its regular elements. Thus $R$ has a right classical quotient ring $Q$. In the above notation we have $a Q+e Q=Q$. Let $x$ be an element of $Q$. Then $x=a c^{-1}$ for some $a, c$ in $R$ with $c$ regular. With $e$ as above we have $x Q=a Q$ and $a Q+e Q=Q$. It follows that $Q$ is von Neumann regular.

(2) Suppose $I=\sum_{i=1}^{n} x_{i} R$. Let $r\left(x_{i}\right)=e_{i} R$ with $e_{i}$ central idempotents. Then $e=$ $e_{1} \cdots e_{n}$ is a central idempotent and $I e=0$. It follows that the sum $I+e R$ is direct. In fact $e R=r(I)$ and $I+e R$ is essential. Also $I+e R$ is generated by the $n$ elements $x_{i}+e$, for if an ideal contains $x_{i}+e$ it also contains $\left(x_{i}+e\right) e=e$ and hence also $x_{i}$.

Proof of Theorem 1. Clearly we have (1) $\Rightarrow(2)$, (3). The implication (2) $\Rightarrow(1)$ follows from Lemma $3(1),[12$, Corollary 2] and [10, Corollary 2], and (3) $\Rightarrow(1)$ follows from Lemma 3(1), [11, Lemma 12(b)] and [10, Corollary 2].

There exists a ring $R$ such that all 2-generated right ideals are projective but $R$ has a nonflat 3-generated right ideal. (See Jøndrup [7, p. 434, Example]. This example was found jointly with P. M. Cohn, as mentioned in [7].) Hence the implications (2) $\Rightarrow(1)$ and $(2) \Rightarrow(3)$ in Theorem 1 are false if one drops the assumption that $R$ is right duo.

We need the following proposition to prove Theorem 2 . Again, we can not remove the right duo hypothesis of $R$. For example, take $R$ to be any simple noetherian non-artinian domain such as the first Weyl algebra, $I$ any nonzero proper right ideal; then $I^{2}=I$ but $I$ is not generated by an idempotent. The question is more interesting perhaps and more relevant for two-sided ideals, and an easy example is to take $R=\left[\begin{array}{lr}\mathbb{Z} & 2 \mathbb{Z} \\ \mathbb{Z} & \mathbb{Z}\end{array}\right] ;$ then $R$ is prime noetherian hereditary; set $I=\left[\begin{array}{rr}2 \mathbb{Z} & 2 \mathbb{Z} \\ \mathbb{Z} & \mathbb{Z}\end{array}\right]$; then $I$ is an idempotent two-sided ideal with zero annihilator so not generated by an idempotent.

Proposition 4. Let $R$ be a right duo ring with a finitely generated idempotent ideal $A$. Then $A=e R$ for some idempotent $e$.

Proof. Let $A=\sum_{i=1}^{n} x_{i} R$. Because $A^{2}=A$, we know that each $x_{i} \in \sum_{i=1}^{n} x_{i} A$. So we get equations of the form

$$
\begin{gathered}
x_{1}\left(1-a_{11}\right)+x_{2} a_{12}+\ldots+x_{n} a_{1 n}=0 \\
x_{1} a_{21}+x_{2}\left(1-a_{22}\right)+\ldots+x_{n} a_{2 n}=0 \\
\ldots \ldots \ldots+\ldots \\
x_{1} a_{n 1}+x_{2} a_{n 2}+\ldots+x_{n}\left(1-a_{n n}\right)=0
\end{gathered}
$$


Since $R$ is right duo, we have $a_{1 n}\left(1-a_{n n}\right)=\left(1-a_{n n}\right) b_{1 n}$ for some $b_{1 n} \in R$, in fact $b_{1 n} \in A$. Now (1) multiplied by $\left(1-a_{n n}\right)$ minus $(n)$ multiplied by $b_{1 n}$ gives

$$
x_{1}\left(1-b_{11}\right)+x_{2} b_{12}+\ldots+x_{n-1} b_{1, n-1}=0
$$

with $b_{1 j} \in A$. Similarly, we get

$$
\begin{gathered}
x_{1} b_{21}+x_{2}\left(1-b_{22}\right)+\ldots+x_{n-1} b_{2, n-1}=0 \\
\ldots \ldots \ldots+\ldots \\
x_{1} b_{n-1,1}+x_{2} b_{n-1,2}+\ldots+x_{n-1}\left(1-b_{n-1, n-1}\right)=0
\end{gathered}
$$

with $b_{i j} \in A$. Continue this until we get $x_{1}\left(1-u_{1}\right)=0$ with $u_{1} \in A$. Using the same method, we have

$$
x_{i}\left(1-u_{i}\right)=0 \quad \text { for all } i \text { with } u_{i} \in A \text {. }
$$

Since $R$ is right duo and $A=\sum_{i=1}^{n} x_{i} R$, we have $A\left(1-u_{1}\right) \ldots\left(1-u_{n}\right)=0$. Let $\left(1-u_{1}\right) \ldots\left(1-u_{n}\right)=1-e$ with $e \in A$. In particular $e(1-e)=0$ so that $e=e^{2}$. Also $A(1-e)=0$; so that $A=R e=e R$, since $e$ is central by [3].

Proof of Theorem 2. If $I$ is, in addition, essential, we shall show that $I$ is invertible.

By Lemma 3(1), $R$ has a right classical quotient ring $Q$ that is von Neumann regular, and then $I Q$ is a finitely generated essential right ideal of the regular ring $Q$. Hence $I Q=f Q$ for some idempotent $f \in Q$. Because $I Q$ is essential, it follows that $I Q=Q$. Hence $I$ contains a regular element of $R$. Also any right $R$-module homomorphism from $I$ to $R$ can be extended to a right $Q$-homomorphism from $I Q$ to $Q$, i.e. from $Q$ to $Q$. Thus we can identify $\operatorname{Hom}_{R}(I, R)$ with the set $I^{*}=\{q \in Q \mid q I \subseteq R\}$. Because $I_{R}$ is finitely generated projective, we have $1 \in I I^{*}$. Let $d$ be a regular element of $R$. Since $R$ is a duo ring, we have $d R=R d$; so $R d^{-1}=d^{-1} R$. Hence $d I^{*} \subseteq R$ if and only if $I^{*} d \subseteq R$. We know that $I$ contains a regular element $c$. Let $I=x_{1} R+\ldots+x_{n} R$. We do not know that the $x_{i}$ are regular. With $r\left(x_{i}\right)=e_{i} R$ as usual, it is easy to show that each $c e_{i}+x_{i}$ is regular and that $I$ is generated by $c$ and the $c e_{i}+x_{i}$. Thus $I$ is generated by regular elements. We have $I^{*} I \subseteq R$, and then $I I^{*} \subseteq R$. Therefore $I I^{*}=R$, and then $I^{*} I$ is an idempotent ideal of $R$. With $I=x_{1} R+\ldots+x_{n} R$, we get $I^{*}=R y_{1}+\ldots+R y_{n}$ for some $y_{i}$. Each $y_{i} x_{j} \in R$; so $R y_{i} x_{j} R=y_{i} x_{j} R$. So $I^{*} I$ is finitely generated by $y_{i} x_{j}$. By Proposition $4, I^{*} I=e R$ for some idempotent $e$. But $I^{*} I$ contains $I$ and so is essential. Therefore $I^{*} I=R$. Hence $I$ is invertible and in particular $I$ is left projective.

Now let $I$ be a finitely generated right projective ideal. By Lemma 3(2), $I$ is a direct summand of a finitely generated essential right projective ideal $K$, say $I+J=K$ with $I+J$ direct. From above $K$ is invertible and left projective. Hence $I$ is left projective.

The next lemma is known, but it is included here since it is unavailable in the literature.

LEMMA 5. Let $M$ be a finitely generated module. If there is an exact sequence $0 \rightarrow N \rightarrow P \rightarrow M \rightarrow 0$, where $N$ is finitely generated and $P$ is finitely related, then $M$ is finitely related.

Proof. Let $f: F \rightarrow P$ be an onto homomorphism, where $F$ is a finitely generated free module with $\operatorname{Ker}(f)$ finitely generated. Assume $N \leq P$ and let $g=\left.f\right|_{f^{-1}(N)}$. We have a 
commutative diagram

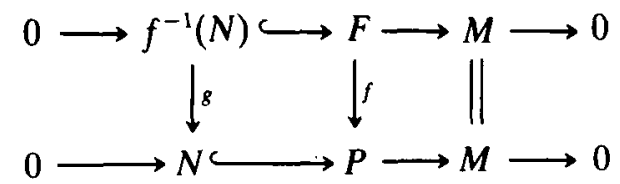

with exact rows, where $g$ is onto, and both $N$ and $\operatorname{Ker}(g)=\operatorname{Ker}(f)$ are finitely generated. So $f^{-1}(N)$ is finitely generated, and hence $M$ is finitely related.

Let $I$ and $J$ be two ideals in a ring. We always have an exact sequence of bimodules

$$
0 \rightarrow I \cap J \stackrel{\alpha}{\rightarrow} I \oplus J \stackrel{\beta}{\rightarrow} I+J \rightarrow 0,
$$

where $\alpha(x)=(x,-x)$ and $\beta((x, y))=x+y$. We shall use this fact without reference.

A ring $R$ is called z.c. (zero commutative) if $l(a)=r(a)$ for all $a \in R$. A reduced ring is $\mathrm{z} . \mathrm{c}$. .

THEOREM 6. Let $R$ be a z.c. duo ring. If every $n$-generated ideal is finitely related as a right module then every $n$-generated ideal is finitely related as a left module.

Proof. The ideal generated by a subset $X \subseteq R$ is denoted by $(X)$. Let $a \in R$. Since $l(a)=r(a)$ and $R$ is duo, ${ }_{R} l(a)$ is finitely generated if and only if $r(a)_{R}$ is finitely generated. It follows that ${ }_{R}(a)$ is finitely related if and only if $(a)_{R}$ is finitely related. This proves the case when $n=1$.

Suppose $n>1$. Now let $I=\left(a_{1}, \ldots, a_{n}\right)$, and assume that $I^{\prime}=\left(a_{1}, \ldots, a_{n-1}\right)$ is finitely related as a left module. We shall show that ${ }_{R} I$ is finitely related.

Since $I_{R}$ is finitely related, the exact sequence of bimodules

$$
0 \rightarrow I^{\prime} \cap\left(a_{n}\right) \rightarrow I^{\prime} \oplus\left(a_{n}\right) \rightarrow I \rightarrow 0
$$

implies that $I^{\prime} \cap\left(a_{n}\right)$ is finitely generated as a right module by Rotman [8, Corollary 3.63]. Therefore $I^{\prime} \cap\left(a_{n}\right)$ is finitely generated as a left module, since $R$ is duo. Now the result follows from Lemma 5.

Corollary 7. A z.c. duo ring is right coherent if and only if it is left coherent.

ACKNOWLEDGEMENT. Both authors would like to thank the referee for many helpful comments, and Xue wishes to express his gratitude to the University of Iowa and University of Maine for their hospitality during the preparation of this paper.

\section{REFERENCES}

1. V. P. Camillo, A note on semi-hereditary rings, Arch. Math. (Basel) 24 (1973), 142-143.

2. S. U. Chase, A generalization of the ring of triangular matrices, Nagoya Math. J. 18 (1961), 13-25.

3. R. C. Courter, Finite dimensional right duo algebras are duo, Proc. Amer. Math. Soc. 84 (1982), 157-161. 106-113.

4. R. Dedekind, Über die Begründung der Idealtheorie, Nachr. Ges. Wiss. Göttingen (1895),

5. S. Endo, Note on p.p. rings, Nagoya Math. J. 17 (1960), 167-170.

6. C. U. Jensen, On characterizations of Prüfer rings, Math. Scand. 13 (1963), 90-98. 431-435.

7. S. Jøndrup, p.p. rings and finitely generated flat ideals, Proc. Amer. Math. Soc. 28 (1971),

8. J. J. Rotman, An Introduction to Homological Algebra (Academic Press, 1979). 
9. L.. W. Small, Semihereditary rings, Bull. Amer. Math. Soc. 73 (1967), 656-658.

10. A. A. Tuganbaev, Distributive modules, Russian Math. Surveys 35(5) (1980), 275-276.

11. A. A. Tuganvaev, Rings with flat right ideals and distributive rings, Math. Notes 38 (1985), 631-636.

12. A. A. Tuganbaev, Hereditary rings, Math. Notes 41 (1987), 173-177.

13. W. V. Vasconcelos, On finitely generated fiat modules, Trans. Amer. Math. Soc. 138 (1969), 505-512.

School of Mathematics

UNIVERSITY WALK

BRISTOL BS8 1TW
Department of Mathematics

UNIVERSITY OF MAINE

Orono, Maine 04469, U.S.A.

Current address for Weimin Xue:

Department OF Mathematics

FujIan Normal University

Fuzhou, FujIan 350007

People's Republic of China 